\title{
New insights on liver carcinogenesis
}

The incidence of human liver cancer is particularly elevated in sub-Saharan Africa and far eastern Asia, where the infections by hepatitis B and hepatitis $\mathrm{C}$ are endemic and food may be contaminated by aflatoxin B1. The incidence is also rising in Europe and United States, due to the elevated occurrence of hepatitis C virus infection, cirrhosis related to type II diabetes, and non-alcoholic steatohepatitis. Liver cancer still is a rapid fatal disease and the therapies with pharmacological agents or alternative strategies do not improve significantly the prognosis of patients with unresectable cancer. This emphasizes the need to investigate the molecular mechanisms responsible for liver cancer development to identify new targets for early diagnosis, chemoprevention, and treatment.

In the last decades a body of work examined the deregulation of signal transduction pathways involved in the pathogenesis of liver cancer, in the attempt to identify novel diagnostic and prognostic markers, and targets for cancer treatment. Several investigators have analyzed the role of aberrant activation of cellular signaling pathways during hepatocarcinogenesis. Novel therapeutic targets were discovered by genome-wide analysis of gene expression leading to the identification of gene signatures of different cancer types. The rationale for the molecular therapy of cancer is the knowledge that oncogenes and oncosuppressor genes encode proteins that are mutated or deregulated forms of key components of regulatory pathways. Thus, in order to kill cancerous cells, leaving unaffected normal cells, huge amount of work attempted to develop anticancer drugs targeting signal transduction mechanisms of liver cells, and a number of signal inhibitors with clinical efficacy and generally favorable toxicity have been introduced into clinical practice.

This special issue of Translational Gastroenterology and Hepatology contains selected review articles on the progress, in the basic and clinical science, of the knowledge on the pathogenesis and molecular therapy of liver cancer, and looks forward to future developments. Several important and timely investigations on various aspects of hepatocarcinogenesis are currently published. Different of these studies are considered in the present special issue. The deregulation of the methionine cycle in liver cancer is presently the object of research by different scientists. The analysis of the role of this deregulation in cancer progression is crucial for its possible links to cancer prognosis and therapy. Other relevant pathogenetic aspects of liver cancer include alterations of mRNA splicing and a spectrum of post-translational modifications of proteins concerning numerous critical signaling events. Indeed, alterations of sumoylation and phosphorylation may influence protein stability and transcription, and protein-protein interactions thus affecting numerous signaling pathways involved in tumor cell growth. Also, the post-translational modifications of proteins may represent a therapeutic approach for liver cancer. Alterations of protein neddylation in HCC have been associated with poor prognosis and a neddylation inhibitor is presently considered for its therapeutic effect.

Branched-chain aminoacids (BCAA) are also the object of recent interesting studies due to their involvement in different bioprocesses including protein metabolism, gene expression, insulin resistance and hepatocytes proliferation. Their importance in liver carcinogenesis is also shown by the observation that the administration of BCAA-rich medicines has positive results in patients with cirrhosis. Studies on the relationships between the level of serum Clusterin and the prognosis of liver cancer revealed that Clusterin could contribute to liver cancer cell migration, epithelial-mesenchymal transition and formation of metastases. The inhibitors of serum Clusterin expression are presently considered for targeted therapy of liver cancer. Other valuable new approaches to the treatment of liver cancer are included in the present special issue. They consider targeting of particular signaling pathways, stimulation of tumor cells autophagy and targeting of these cells and their permissive microenvironment simultaneously.

This special issue of the Translational Gastroenterology and Hepatology has not been conceived to propose a comprehensive overview of all recent advances in the pathogenesis and personalized molecular therapy of liver tumors. The aim of this issue is to focus on some of the major topics, treated by experts in the field, dedicated to liver cancer prevention and therapy. The lesson for future developments is the need to expand our knowledge on the deregulation of cellular signaling pathways, involved in liver cancer development and progression, in order to identify new "druggable" targets. Furthermore, the effects of the combined inhibition of different signaling pathways, the correlations between molecular deregulation and response to therapy, and the methods to select the patients that could be successfully treated by personalized therapy are major themes 
treated in the present special issue, which can hopefully provide stimulus for further advances in the field.

\section{Acknowledgements}

None.

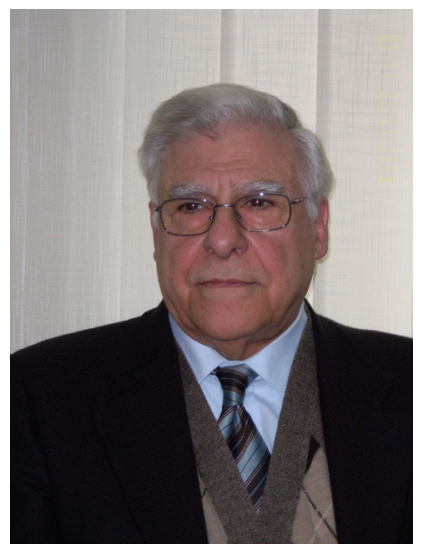

Francesco Feo

Francesco Feo, MD

Professor Emeritus of Experiential Pathology, Department of Clinical, Surgical and Experimental Medicine, Division of Experimental Pathology and Oncology, University of Sassari, Sassari, Italy. (Email: feo@uniss.it) doi: $10.21037 /$ tgh.2018.07.14 Conflicts of Interest: The author has no conflicts of interest to declare. View this article at: http://dx.doi.org/10.21037/tgh.2018.07.14

doi: $10.21037 / \operatorname{tgh} .2018 .07 .14$

Cite this article as: Feo F. New insights on liver carcinogenesis. Transl Gastroenterol Hepatol 2018;3:50. 